\title{
A Novel Mean Median Filter for Noise and Artifacts Suppression from Digital Images
}

\author{
Geeta Hanji \\ PDA Engg.College, \\ Gulbarga, India
}

\author{
M.V.Latte \\ JSSIT \\ Bangalore, India
}

\begin{abstract}
A novel mean-median filter is proposed for the suppression of impulse noise and various artifacts from the digital images. Leading Diagonal Sorting Algorithm is used with the fixed $3 \times 3$ size working window to compute the median. Truncated mean is computed by defining the boundaries and truncating the pixel values in the filtering window that fall outside the defined boundary. Noise detection is carried in two steps: In the first step the 'reference pixel' is tested for the presence of impulses with Min-Max detection strategy. In the second stage of detection, edge preserving unique criteria is employed to further classify the pixel under test as 'noisy' or 'edge belonged'. This intelligent edge preserving decision criterion decides whether the test pixel deserves the restoration or not and facilitates the restoration of the noisy pixel either by the 'truncated mean' or by the 'window median' based on the decision threshold. Performance of the proposed filter algorithm is studied on a large number of images with varying amounts of salt and pepper noise and several types of artifacts and their combinations. Simulation results prove that the proposed algorithm (PA) effectively suppresses the high density salt and pepper noise (SPN) and in addition, it performs excellent in suppressing image artifacts such as strip lines (both white and black), drop lines (both white and black), missing bands and noise blotches. The novelty of the proposed mean-median filter lies in the fact that it attempts to simultaneously suppress impulsive noise and artifacts with good edge preservation (as the maximum size of the filtering window is restricted to ' $3 \times 3$ ' only) and also because a two stage detection mechanism is employed. The goodness of the proposed algorithm lies in replacing several independent filter schemes required for suppression of noise and artifacts of several types without much distorting the vital features of the image under test. The performance evaluation of the proposed algorithm is done in terms visual appearance and quantitative measures such as Mean Square Error (MSE), Peak Signal to Noise Ratio (PSNR), Image Enhancement Factor (IEF) and the Computation Time (CT). Simulation results are compared with other state of art algorithms to derive the meaningful conclusions.
\end{abstract}

\section{General Terms}

Image Processing, Noise and Artifacts Suppression.

\section{Keywords}

Noise, Artifacts, Sorting, Truncated Mean, Impulse noise, Strip Lines, Blotches, Drop lines, Missing bands, PSNR, MSE, Run time, IEF.

\section{INTRODUCTION AND LITERATURE SURVEY}

Very often digital images get degraded by several types of noises and artifacts due to low quality image sensors or transmission medium errors [1]. Salt and pepper noise (also called fixed value impulse noise), additive noise and random noise are the major and generally occurring noise types with the digital images during acquisition, transit, storage and retrieval [2]. In applications like remote sensing, along with the impulsive noise, artifacts such as drop lines, white and black strip lines, missing bands and blotches occur [3]. However, in several image processing operations and in multimedia applications much attention is given towards achieving the processed images with the pleasing and flawless visual defects along with more amount of details and other vital features preserved intact. In the literature are reported a large number of methods to get rid of above mentioned noise types with an effort to achieve better results. However very few numbers of research papers are available on image artifacts suppression such as drop lines, missing bands etc., which occur along with the impulse noise [3] in applications such as satellite imagery. A brief survey and details of the reported work in the field of image noise reduction and artifacts suppression is presented below:

As mentioned above, in applications like remote sensing, along with the impulsive noise, artifacts such as drop lines, white and black strip lines, missing bands and blotches occur [3].Degradations of impulsive nature which posses' shapes of irregularity and are randomly spread over the entire image are noise blotches. A remedy for removing the blotches in images was proposed by Silva and Corte-Real et al [4].Authors state that bright and dark scratches affect positive and negative films respectively. Variations in brightness of adjacent lines results in artifacts known as strip lines [3].Unequal responses of elements of a detector array to the same amount of incoming electromagnetic energy is the main cause for these variations. Strip lines were considered as line scratches for sequences of images in the proposed work by Silva and Corte-Real [4]. Kokaram [5] has proposed a remedy for removal of scratches based on temporal filtering in image sequences.

When a detector does not work properly for a short duration of time, artifacts known as drop lines occur and the work presented by Shahrokhy [3] addressed the issue of suppression of drop lines. Silva and Corte-Real [4] consider drop lines as line scratches for the sequences of images in their presented work.

Another severe artifact and a serious problem is band missing [3], [4]. Band missing is caused by the corruption of two or more drop/strip lines continuously. Based on the temporal filtering, Kokaram [5] has proposed a remedy for the restoration of missing data in the image sequences.

Narrow vertical, bright/dark lines that affect a column or a set of columns of the image are referred to as scratches. Strip lines and drop lines were considered as line scratches in the method proposed by Silva et al [3]. Bright scratches are found in positive type film and dark scratches are found in negative films. Milady [4] addressed only dark scratches and occurrence of bright scratches were inverted and the same 
algorithm was used to address the problem. Along with the artifacts such as blotches, drop lines etc., in IP applications impulsive noise also appears and causes image degradations. As a result, image appears degraded [1] and presents itself with an unpleasant and flawed look while masking several important fine details such as edges, fine lines, texture and other vital features of the underlying image. Thus the overall performance of the system degrades continuously as the noisy images affect the performance of the subsequent blocks of processing in the image processing chain. Hence noise and artifacts suppression plays an important role in the field of digital image processing. Also it is necessary to suppress the impulsive noise and artifacts occurring in the images with a single and computationally efficient algorithm. However the literature review reveals that, very less amount of work has been carried out in attempting to reduce image artifacts along with the impulsive noise in image processing fields such as remote sensing etc.

However a lot of research has been carried out in the field of image noise cleaning with detail preservation by different researchers. A detailed survey of various methods reported in the literature to suppress several types of noise that gets added with the images during acquisition, transit, storage can be found from [6],[7],[8],[9],[10]. Since the work presented in this paper basically deals with the suppression of fixed valued impulsive noise along with the artifacts like drop lines, noise blotches etc., few of the important works reported in the literature are as below:

Manikandan [3] proposed a decision based adaptive median /mean filter algorithm that can simultaneously remove impulses, strip lines, drop lines, band missing and blotches while preserving edges from still images. He extended the same technique to image sequences (video) by incorporating techniques for motion compensation. The advantage of the proposed algorithm is that a single algorithm with improved performance can replace several independent algorithms required for removal of different artifacts. However the proposed method performed well in suppressing noise up to $60 \%$ density in the presence of low amounts of artifacts and suffered edge blurring. Vijakumar [11] proposed a decision based, nonlinear, adaptive, median filter to remove random valued impulse noise (RVIN) with artifacts such as streaks, stripes, blotches based on ROAD statistics. Proposed algorithm is simple, can be implemented in hardware, computationally fast, but suffers from a limitation of suppressing noise along with artifacts only up to $60 \%$. Suresh Kumar and Mangalam [12] proposed an adaptive based artifacts removal algorithm for the removal of blocking artifacts, blotches, impulses, drop lines and strip lines in images. Detected noise variance level is considered as the main indicator in their work to choose the proper method for restoration. An adaptive window is used during the filtering operation depending on noise level. Proposed method attempted to replace the number of independent algorithms required for removal of blocking and other different artifacts while giving better results. The results proved that the algorithm is more efficient in suppressing the effects of strip lines, drop lines, blotches along with impulse noise of varying noise densities up to $80 \%$. However their method did not consider the image sequences. Satpathy, Nagwanshi, Nayak and Ardil [13] proposed a decision based, 2-stage nonlinear algorithm for suppressing drop lines, band lines, band lost and impulses in images. Artifacts removal is achieved without damaging edges and details. Whenever noise is excessive, the proposed algorithm automatically switches to mean filtering. Proposed algorithm is advantageous in the sense that it can replace several independent algorithms for the removal of different artifacts. Vasant and Jawahar [14] proposed a fixed $3 \times 3$ window and threshold based, non linear filter for the removal of image artifacts such as black band noise, blotches, streaks and impulse. The proposed algorithm is non adaptive and can replace several individual algorithms for the removal of different image artifacts and presented good results in eliminating fixed value impulse noise up to $70 \%$, but introduces blurring at very high noise densities. Geeta, Latte and Shweta [15] attempted to improve the method proposed by Manikandan [3] by proposing a new threshold value for the detection of noisy pixels and were able to obtain little improved results. Mahesh and Tamilselvam [16] addressed the issue of noise and artifacts removal from color images using the method proposed by [15] Geeta, Latte and Shweta. However the results did not prove satisfactory performance at higher noise densities. Kiruthika and Sharat Babu[17] proposed a novel and effective method for removal of blotches and impulse noises in corrupted color images based on a two stage method in which, the first stage is a noise detection phase where a nonlinear decision based algorithm is used to detect impulse noise pixels. The second is a noise filtering phase where a new algorithm based on performing vector median in RGB is used. In their work they used a decision threshold value suggested by Geeta, Latte and Shweta [15]. The results of simulations show that the proposed method is capable of detecting all the impulse noise pixels with almost zero false positive rates and removes noise while retaining finer image details. It outperforms the standard procedures and is yet simple and suitable for real time applications, but its better performance is limited only up to $60 \%$ noise density and low amounts of artifacts.

From the review of literature, it can be concluded that each of the above mentioned proposals suffer its own flaws and weaknesses. Many of them [15], [16] could not address suppression of impulsive noise of more than 50\% density along with various artifacts. Methods proposed in [14] and [11] did not consider the most commonly occurring fixed value impulsive noise. Literature review also reveals the fact that a class of basic non linear filter (median filter) and its several variants, modified and enhanced versions have proved to produce best results as these are capable of providing better suppression of impulsive noise without losing the information about edge and other vital features. Thus there exists a lot of scope to develop and explore nonlinear decision based filter algorithms for addressing various noise types and image artifacts with a single, common algorithm. Hence the main objective of this paper is to propose an adaptive threshold, nonlinear decision based mean-median filter algorithm to suppress several artifacts along with the impulse noise while preserving the image edges. In this regard, the work proposed and presented in this paper under the title "A Novel MeanMedian Filter for Noise and Artifacts Suppression from Digital Images" is a novel (i.e. adaptive threshold based, nonlinear, decision based, edge preserving, exploiting the merits of both the mean and median filters and computationally efficient) mean-median filter. An application of nonlinear decision based mean-median filter algorithm for the excretion of high density impulses, strip lines, drop lines, missing bands, and noise blotches with a good edge preserving capabilities has been developed and presented in this paper. The advantage of the proposed algorithm is that a single algorithm with improved performance can replace several independent algorithms required for removal of different artifacts along with the impulsive noise. 
Organization of this paper is as follows. Section1 deals with the introduction and review of the literature. Section 2 discusses the proposed algorithm (PA) to remove SPN and artifacts along with the suitable illustrations. Section 3 presents and compares the results of the proposed work with other state of techniques and the conclusions are derived in section 4 highlighting the scope for future work.

\section{PROPOSED ALGORITHM}

\subsection{Outline of the proposed Algorithm}

The proposed mean-median based filter algorithm consists of the following steps:

Step 1: A $3 \times 3$ filtering window ' $\mathrm{W}(\mathrm{x}, \mathrm{y})$ ' is chosen with the pixel to be processed ' $\mathrm{X}(\mathrm{i}, \mathrm{j})$ ' at the centre.

Step 2: The test pixel ' $\mathrm{X}(\mathrm{i}, \mathrm{j})$ ' is tested for the condition $0<\mathrm{X}(\mathrm{i}, \mathrm{j})<255$ and is declared as an 'uncorrupted pixel' if this condition is true and its value is unaltered.

Step 3: If X (i,j) holds ' 0 ' or ' 255 ' then it is further processed as follows:

3a) If test pixel ' $X(i, j)$ ' is ' 0 ' ( or $X_{\text {min }}$ ), then number of occurrences of ' 0 ' are counted from all the remaining unprocessed pixels.

3b)Similarly if the test pixel is ' 255 ' (or $X_{\max }$ ), then number of occurrences of ' 255 ' is counted from all the remaining unprocessed pixels.

In both the cases (i.e. with a $3 \times 3$ filtering window) there are four unprocessed pixels. We let the count of zero's (0's) or one's (1's) as ' $\mathrm{C}$ ' in both the cases. Now two different cases arise w.r.t. the value of count ' $\mathrm{C}$ '.

Case i): If $\mathrm{C}<3$, then the 'reference center pixel' is regarded as a noisy pixel and is replaced with an appropriate substitute (say ' $\boldsymbol{\beta}$ ') which may be the window median ' $\mathrm{M}(\mathrm{d})$ ' or truncated Mean $\mathrm{M}(\mathrm{t})$ which will be discussed in step 6 of section 2.2 .

Case ii): If $\mathrm{C} \geq 3$, then the 'reference (or 'test') pixel' is regarded as a pixel belonging to the image edge, and is left undisturbed.

Step 4: Steps 1 to 3 are repeated for all the pixels in the image (i.e. for the whole image).

\subsection{Computation of 'Truncated Mean M (t)' and Decision Threshold ' $T$ (h)'.}

Step 1) Compute the arithmetic average of all nine pixels in the filtering window, i.e. $\mathrm{M}(\mathrm{a})=\operatorname{Average}(\mathrm{X})$.

Step 2) Compute the decision threshold $\mathrm{T}(\mathrm{h})$ as follows :

$\mathbf{T}(\mathbf{h})=\frac{1}{M \times N} \sum_{i=1}^{M} \sum_{j=1}^{N} \mid X(i, j)-M(a)$

Step 3) Now truncate the pixel values in the vector $X=$ $\{X(i, j)\}$ by,

$\{\mathbf{X}(\mathbf{i}, \mathbf{j})\}=\left\{\begin{array}{l}M(a)+T(h) ; \text { if } X(i, j)>M(a)+T(h) \\ M(a)-T(h) ; \text { if } X(i, j)<M(a)-T(h)\end{array}\right.$

where $1 \leq(\mathrm{i}, \mathrm{j}) \leq \mathrm{M} \times \mathrm{N}$ and $\mathrm{M} \times \mathrm{N}$ is window size[18] .

Step 4) Now compute the truncated mean $M(t)$ as,

$$
\mathrm{M}(\mathrm{t})=\text { Mean }\{\mathrm{X}(\mathrm{i}, \mathrm{j})\} .
$$

Step 5) Find the window median $M(d)$ using Leading Diagonal Sorting Algorithm [19].
Step 6) Now it is required to find which one the above $(\mathrm{M}(\mathrm{t})$ or $\mathrm{M}(\mathrm{d}))$ is nearer to the center of the interval $\left(X_{\min }, X_{\max }\right)$. Hence we define [23],

$$
\begin{aligned}
& D_{1}=\left(X_{\max }+X_{\min }\right)-2 M(t) \\
& \text { and } \quad D_{2}=\left(X_{\max }+X_{\min }\right)-2 M(d) \\
& \text { If } D_{1}>D_{2} \text {, then } \beta=M(t) ; \text { otherwise } B=M(d) \text {. }
\end{aligned}
$$

\subsection{Leading Diagonal Sorting Algorithm}

In the computation of filtering window median, basic operation and a key step involved is sorting the elements belonging to the current working window. Various sorting algorithms such as bubble sort, binary sort, merge sort, shear sort, quick sort etc. are available. Many of these algorithms require more comparators and hence are less computationally efficient. For real time applications, computationally efficient algorithms are needed. The work presented in this paper uses Leading Diagonal Sorting Algorithm (LDSA) proposed and used by Rajmani and Keshveni [19] in which the sorting of the leading diagonal elements alone is done rather than traditional row, column and diagonal sorting as a whole. In this method only 3 pixels are involved for sorting and both the computational and hardware complexity are reduced to a larger extent. Computation of the filtering window median $\mathrm{M}(\mathrm{d})$ using leading diagonal sorting is illustrated as shown in figures 1 and 2 . The algorithm is explained with the following steps:

Step1: A sliding window of $3 \times 3$ in a $512 \times 512$ size standard 'LENA' image is considered.

Step2: Elements of the leading diagonal are sorted in ascending order.

Step3: The intensity values pixels are compared against salt and pepper noise ( 0 or 255 ) affected pixels and then replaced with either of the other two pixel values that is noise free.

Step4: Step 3 is repeated for the whole image, by sliding the working window to the next position.

\section{EXPERIMENTAL RESULTS AND DISCUSSION}

The proposed algorithm is tested on images affected with salt and pepper type impulsive noise and artifacts such as strip lines (both dark and bright), drop lines (both dark and bright), noise blotches and missing bands. The results are compared with those of standard median filter (SMF) [1], DBA [20], MDBUTMF [21], [3], [11]. A quantitative comparison is done in terms of MSE, PSNR, IEF and computational time of the algorithms. Performance of various algorithms at different SPN for PSNR measure are illustrated with the aid of a bar chart in figure [3]. Figure 4 shows the graphical representation of comparison results of computation time. Figure 5 illustrates the IEF results comparison of the proposed filter with the other competitive filters. Table 3 illustrates the overall comparison results of PA with various other filters in terms of PSNR, MSE, IEF and CT in seconds for the images corrupted with $20 \%$ and $40 \%$ SPN and various image artifacts such as strip lines, drop lines etc. From the results it is evident that, the performance of the proposed algorithm is much better than the most competitive state of art filters like those reported in references [14], [20], [21] etc. This argument is also supported by the set of figures shown in 6,7, 8,9 and 10 for different images contaminated with various types of degradations along with SPN. In figures 10 and 11 
the Pout.tif and cell.tif images are corrupted with 50\% SPN and various artifacts. For all the situations, the proposed scheme performs well in denoising and artifacts reduction with a single competitively efficient filter. Thus the superior performance of the proposed algorithm over the other state of art filters is realized in terms of the pleasing and information rich images, in the sense that the image edges are well preserved as seen in all the simulation results.

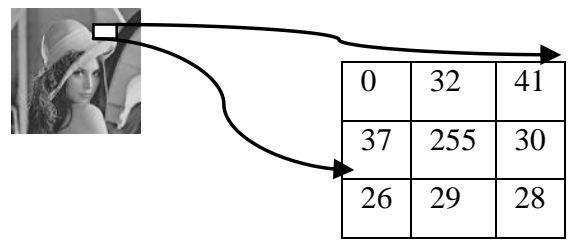

Figure 1. A small portion of Lena image showing 9 pixels in a $3 \times 3$ window.

\begin{tabular}{|c|c|c|c|c|c|c|c|c|c|c|c|}
\hline 0 & 32 & 41 & $Q$ & 32 & 41 & 28 & 32 & 41 & 28 & 32 & 0 \\
\hline 37 & $\lambda$ & 30 & 37 & 2 & 30 & 37 & 2 & 30 & 37 & 28 & 30 \\
\hline 26 & 29 & 28 & 26 & 29 & 255 & 26 & 29 & 255 & 26 & 29 & 28 \\
\hline
\end{tabular}

(a)

(b)

(c)

(d)

Figure 2.(a)Leading diagonal elements (LDE) 0,255,28 (b) Sorted LDE (c) After replacement of noisy pixel '0' (d)After replacement of noisy pixel ' 255 ' and final $3 \times 3$ filtered (or de-noised) window.

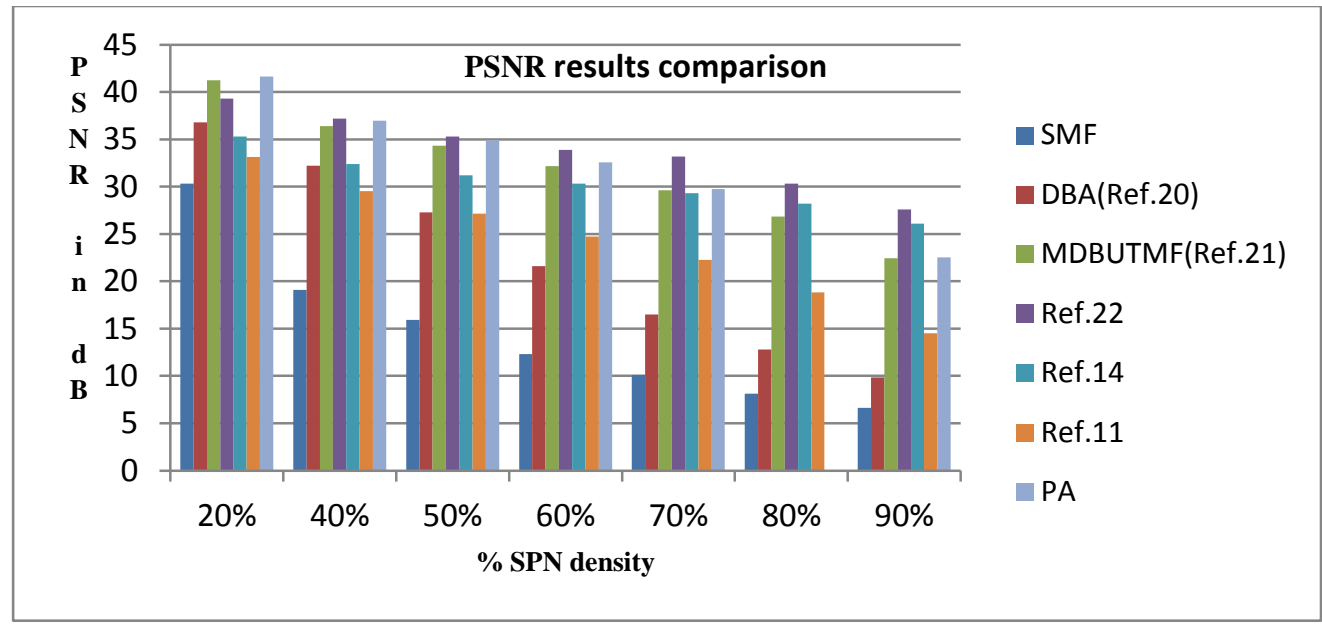

Figure 3. PSNR results comparison of PA with various other filters for the images noised with varying amounts of SPN.

\begin{tabular}{|c|c|c|c|c|c|c|}
\hline 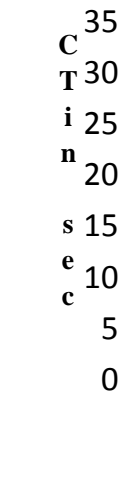 & 20\% 50\% 70\% 90\% & $\begin{array}{l}\text { esults } \\
- \text { SBF } \\
- \text { MDB(Ref.20) } \\
\text {.21) } \\
* \text { Ref.22 } \\
\approx \text { PA }\end{array}$ & $\begin{array}{l}\mathbf{I} \\
\mathbf{E} \\
\mathbf{F}\end{array}$ & $\begin{array}{r}350 \\
300 \\
250 \\
200 \\
150 \\
100 \\
50 \\
0\end{array}$ & $\begin{array}{c}50 \% \quad 60 \% \quad 70 \% \quad 80 \% \quad 90 \% \\
\% \text { SPN density }\end{array}$ & $\begin{array}{l}\approx \text { SMF } \\
- \text { DBA(Ref.20) } \\
- \text { Ref.21 } \\
\approx \text { Ref.14 } \\
\approx \text { PA }\end{array}$ \\
\hline $\begin{array}{r}\text { Fig.4 RuI } \\
\text { th }\end{array}$ & $\begin{array}{l}\text { n time results comparison } \\
\text { e images corrupted with }\end{array}$ & $\begin{array}{l}\mathrm{A} \text { and other filters for } \\
\text { ind strip lines. }\end{array}$ & $\begin{array}{l}\text { Fig } 5 . \\
\text { corrup }\end{array}$ & $\begin{array}{l}\text { IEF } \\
\text { ted w }\end{array}$ & $\begin{array}{l}\text { results comparison of PA with ot } \\
\text { ith SPN and strip lines. }\end{array}$ & er filters for the images \\
\hline
\end{tabular}

Table 3. Performance of P.A and various algorithms at $20 \%$ and $40 \%$ SPN + various artifacts for Lena image 


\begin{tabular}{|c|c|c|c|c|c|c|c|c|c|c|c|c|}
\hline \%ND & \multicolumn{3}{|c|}{ PSNR(dB) } & \multicolumn{4}{|c|}{ MSE } & \multicolumn{3}{c|}{ IEF } & \multicolumn{4}{c|}{ CT( Sec) } \\
\hline & Rf.3 & Rf.14 & PA & Rf.3 & Rf.14 & PA & Rf.3 & Rf.14 & PA & Rf.3 & Rf.14 & PA \\
\hline $20 \%$ & 26.60 & 30.89 & 32.24 & 1419 & 808 & 773 & 36.89 & 41.90 & 43.45 & 30.26 & 29.34 & 14.82 \\
\hline $40 \%$ & 25.87 & 29.02 & 30.98 & 1643 & 985 & 921 & 33.32 & 39.65 & 42.33 & 33.35 & 25.02 & 19.08 \\
\hline
\end{tabular}
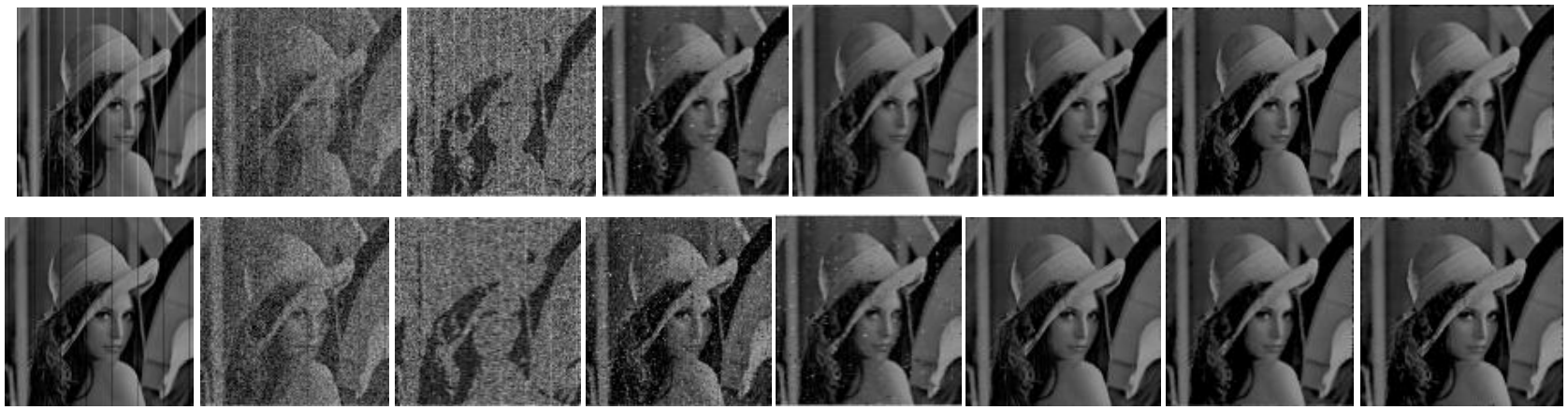

Figure 6. (a)Original Lena (b)Lena+bright (and dark) in upper (and lower) figures (c) Lena +Strip lines+50\% SPN (d) Binary flag image (e) SMF (5X5) (f) Ref.3 (g) DBA (Ref.20) with threshold=60 (h) DBA with t=50. (i) PA
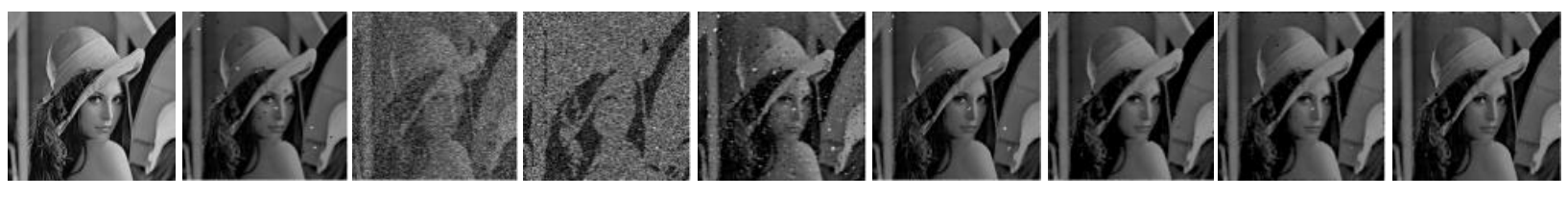

Figure 7. (a) Original Lena. Image with (b)(bright)Blotches (c) Blotches $+50 \%$ SPN (d)Binary flag image. Results of (e)SMF(5X5) (f) Ref.3 (g) Ref.20 with t=60. (h)Ref. 20 with $\mathrm{t}=50$. (i) Proposed Algorithm (PA).

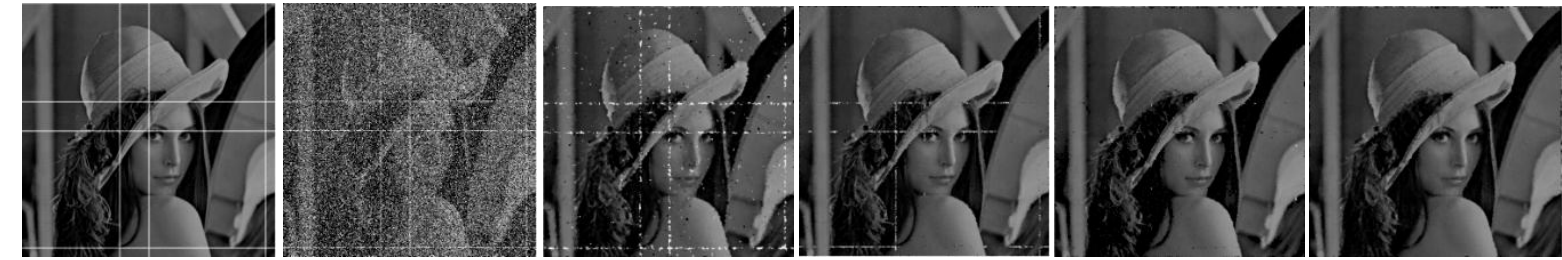

Figure 8. Missing band $+50 \%$ SPN removal Lena with a) missing bands. b)Missing bands $+50 \%$ SPN. Results of c) SMF d) Ref.3. e) Ref.15. f) PA.
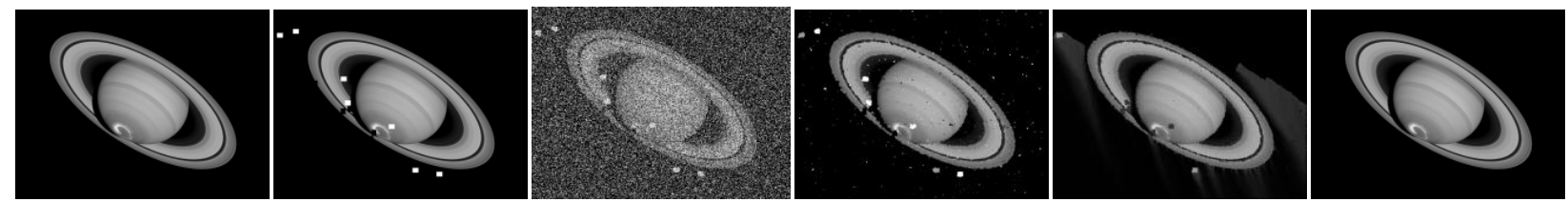

Figure 9. (a) Original Saturn Image. Image with (b)Blotches (c)Blotches+ 50\% SPN (d) SMF(5×5) (e) Ref (3) (f) Proposed
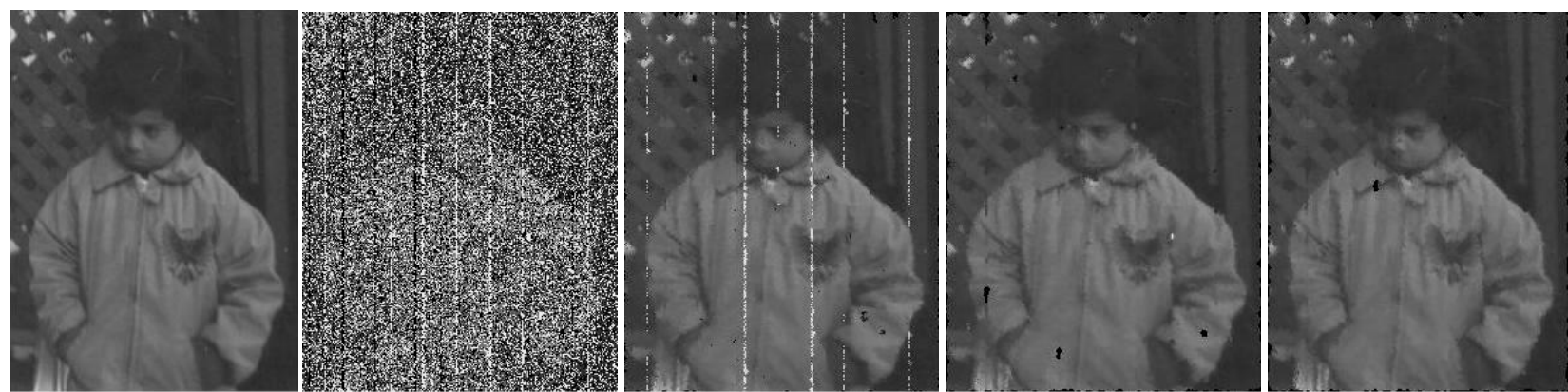

Figure 10. 50\% SPN +All artifacts suppression a)Pout.tif (original) b)Pout with 50\%SPN +All other artifacts . Results of c) Ref. 3. d) Ref.15. e)PA 

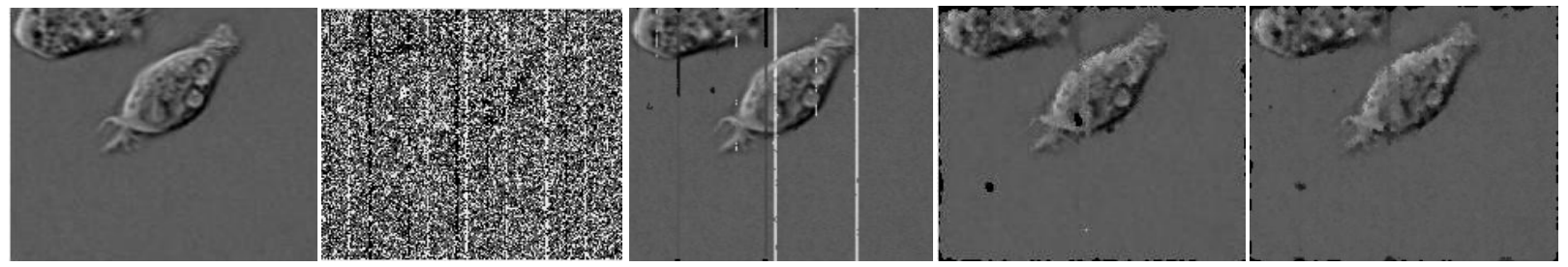

Figure 11. 50\% SPN +All artifacts suppression (a) Cell.tif (Original) b) Ceil with 50\% SPN +All artifacts. Results of c) Ref.3, d) Ref.15, e) Proposed method

\section{CONCLUSIONS}

A novel mean-median filter is proposed for the suppression of impulse noise and various artifacts from the digital images. We have combined the advantages of standard and state-ofthe-art methods to achieve high results in terms of PSNR and IEF. Furthermore, this method has reasonable computation time in high noise densities (CT).Leading Diagonal Sorting Algorithm[19] is used with the fixed $3 \times 3$ size working window to compute the median. Truncated mean is computed by defining the boundaries and truncating the pixel values in the filtering window that fall outside the defined boundary. Noise detection is done with an intelligent edge preserving decision criterion. The main contribution of this paper is the design of a simple and strong impulse noise detection method using an adaptive decision threshold. The method is very simple, easy to implement, and computationally an efficient one. Performance of the proposed filter algorithm is studied on a large number of images with varying amount of salt and pepper noise and several types of artifacts and their combinations. Simulation results show that the proposed method performs better than some of the existing methods, both visually and quantitatively, giving acceptable and recognizable restored images by preserving their vital features at medium to high noise levels. Although, the performance of the proposed method is not the best, but works reasonably well compared to some of the popular methods mentioned earlier. The performance of the proposed method may be improved by using robust estimation techniques. Rigorous study is required to fix better threshold value which will help further in improving the performance of the proposed method. Proposed algorithm can be further improved by using different noise detectors and efficient filters that are suitable for Gaussian and random valued impulsive noise (RVIN) and also for the application in video.

\section{ACKNOWLEDGEMENTS}

Thanks to the reviewers for providing the valuable feedback to improve the quality of the manuscript, especially with respect to the discussion of results and providing an extra space to incorporate their suggestions.

\section{REFERENCES}

[1] A. Bovik, Handbook of Image and Video Processing, Academic Press, 2000.

[2] S.M.Shahrokhy,Visual and Statistical Quality Sssessment and Improvement of Remotely Sensed Images, Proceedings of the 20th Congress of the International Society for Photogrammetry and Remote Sensing (ISPRS'04), Turkey, July 2004.

[3] S.Manikandan and D.Ebenezer,A Nonlinear Decisionbased Algorithm for Removal of Strip lines, Drop lines, Blotches, Band missing and Impulses in images and videos. Proceedings of EURASIP Journal on Image and Video Processing, Article ID 485921, 2008.
[4] A.U.Silva and L.Corte-Real, Removal of Blotches and Line scratches from Film and Video Sequences using a Digital Restoration Chain, Proceedings of the IEEEE URASIP workshop on Nonlinear Signal and Image Processing (NSIP ‘ 99),pp.826- 829,Antalya,Turkey,June 1999.

[5] A.Kokaram, Detection and Removal of Line Scratches in Degraded Motion Picture Sequences, Proceedings of the $8^{\text {th }}$ European Signal Processing Conference (EUSIPCO' 96), vol.1, pp.5-8, Trieste, Italy, September 1996.

[6] Sonali R. M, Nileshsingh V.T., A Comparative Study of Image Filtering on Various Noisy pixels, International Journal of Image Processing and Vision Sciences, ISSN (Print): 2278 - 1110, Volume-1, Issue-2, 2012.

[7] Sin Hoong Teoh, Boon Tatt Koik, and Haidi Ibrahim, Exploration of Current Trend on Median Filtering Methods Utilized in Digital Grayscale Image Processing, International Journal of Materials, Mechanics and Manufacturing, Vol. 1, No. 1, February 2013.

[8] Geeta Hanji and M.V.Latte, A New Threshold Based Median Filtering Technique for Salt and Pepper Noise Removal,(ICDIP-2010), 2010 Proceedings of SPIE, Vol.7546, PP754639-754639-10,Singapur, 2010, DOI: 10.1117/12.856333. http://dx.doi.org/10.1117/12.856333.

[9] Geeta Hanji and M.V.Latte, Detail Preserving Fast Median Based Filter," Journal of Advanced Computer Science and Technology, 1(4) (2012) 195-206 ( S Science Publishing Corp oration,www.sciencepubco.com/index.php/JACSTarticle /view

[10] Geeta Hanji, M.V.Latte, A New Impulse Noise Detection and Filtering Algorithm, Image Processing \&Communications Life Sciences, ISSN (Print): $2278-$ 1110, Volume-1, Issue-2, 2012, IPC, The Journal of University of Technology and Life Sciences in Bydgoszcz, Vol. 16, no.1-2, pp.43-48, DOI: 10.2478/v10248-012-0004-4.

[11] V. R. Vijaykumar, P. Jothibasu, Decision based adaptive median filter to remove blotches, scratches, streaks, stripes andimpulse noise in images. Proceedings of IEEE Int. conference on Image Processing , pp. 117-120, Sep 2010.

[12] Suresh, Mangalam, Removal of different artifacts and impulse noise in grey images, Journal of Theoretical and Applied Information Technology ISSN: 1992-8645.

[13] S. K. Satpathy, S. Panda, K. K. Nagwanshi, S. K. Nayak and C. Ardil, Adaptive Non-linear Filtering Technique for Image Restoration International Journal of Electrical and Computer Engineering 5:1 2010.

[14] K.Vasanth,V.Jawahar senthilkumar, Novel Scheme for removal of Blotches, Black bands, Streaks and Impulse 
Noise in grey images, Indian Journal of Computer Science and Engineering (IJCSE), ISSN : 0976-5166 Vol. 3 No.4 Aug-Sep 2012.

[15] Geeta Hanji, M.V.Latte, N. M. Shweta , An Improved Nonlinear Decision Based Algorithm for Removal of Blotches and Impulses in Gray scale Images. Proceedings of the Second International Conference,ACCT,ROHTAK,Haryana.

[16] P.Tamilselvam, M.V.Mahesh and G.Prabhu,Blotches and impulse removal in colour scale images using non-linear decision based algorithm, International journal of scientific research publications,vol.3, no.4, pp.1-5, 2013.

[17] Kiruthika. D \& Sarathbabu. R An Improved Nonlinear Decision based Algorithm for Removal of Blotches and Impulses in Color Images, International Journal of Advanced Electrical and Electronics Engineering, (IJAEEE) ISSN (Print) : 2278-8948, Volume-2, Issue-3, 2013.

[18] Xudong Jiang, Iterative Truncated Arithmetic Mean Filter and its Properties, Transactions on Image Processing, Vol. 21, No. 4, April 2012.

[19] A.Rajamani, V.Krishnaveni, Impulse Denoising Algorithm for Gray and RGB Images, International
Journal of Computer Applications (0975 - 8887) Volume 70- No.2, May 2013.

[20] Nair, Rajgiri, Revathy, Tatavarti, An Improved Decision-Based Algorithm for Impulse Noise Removal, Congress on Image and Signal Processing, 2008. CISP 08. (Volume: 1) 426- 431, Print ISBN: 978-0-76953119-9, IEEE publisher.

[21] S.Esakkirajan, T.Veerakumar, Adabala, Removal of High Density Salt and Pepper Noise Through Modified Decision Based Unsymmetrical Trimmed Median Filter, IEEE Signal Processing Letters, Vol. 18, No.5, May 2011. http://dx.doi.org/10.1109/LSP.2011.2122333.

[22] Vasant, Jawahar, A Decision based Trimmed Midpoint Algorithm for the Removal of High Density SPN Journal of Theoretical and Applied Information Technology, August 2012. Vol. 42 No.2 () 2005 - 2012 JATIT \& LLS. ISSN: 1992-8645 .E-ISSN: 1817-3195.

[23] A. Jourabloo , A.H. Feghahati, M. Jamzad, New algorithms for recovering highly corrupted images withImpulse noise, Scientia Iranica Transactions D: Computer Science \& Engineering and Electrical Engineering Scientia Iranica D (2012) 19 (6), 17381745 . 